\title{
A single use electrochemical sensor based on biomimetic nanoceria for the detection of wine antioxidants
}

Veronica Andrei, ${ }^{\dagger}$ Erica Sharpe,${ }^{ \pm}$Alina Vasilescu,${ }^{* \dagger}$ Silvana Andreescu ${ }^{ \pm *}$

${ }^{\dagger}$ International Centre of Biodynamics, 1B Intrarea Portocalelor, 060101 - Bucharest, Romania

${ }^{ \pm}$Clarkson University, Department of Chemistry and Biomolecular Science, Potsdam, NY 136995810 .

*Corresponding authors: avasilescu@biodyn.ro; eandrees@ clarkson.edu 


\begin{abstract}
We report the development and characterization of a disposable single use electrochemical sensor based on the oxidase-like activity of nanoceria particles for the detection of phenolic antioxidants. The use of nanoceria in the sensor design enables oxidation of phenolic compounds, particularly those with ortho-dihydroxybenzene functionality, to their corresponding quinones at the surface of a screen printed carbon electrode. Detection is carried out by electrochemical reduction of the resulting quinone at a low applied potential of $-0.1 \mathrm{~V} v s$. the $\mathrm{Ag} / \mathrm{AgCl}$ electrode. The sensor was optimized and characterized with respect to particle loading, applied potential, response time, detection limit, linear concentration range and sensitivity. The method enabled rapid detection of common phenolic antioxidants including caffeic acid, gallic acid and quercetin in the concentration range from 1 to $100 \mu \mathrm{M}$, and demonstrated good functionality for the analysis of antioxidant content in several wine samples. The intrinsic oxidase-like activity of nanoceria shows promise as a robust tool for sensitive and cost effective analysis of antioxidants using electrochemical detection.
\end{abstract}

Keywords: enzyme mimetic; nanoceria; oxidizible phenolics; wine antioxidants; disposable sensors; amperometry 


\section{Introduction}

Cerium oxide nanoparticles $\left(\mathrm{CeO}_{2} \mathrm{NPs}\right)$ or nanoceria particles have received significant attention due to their unique catalytic and free radical scavenging properties [1] which make them attractive for a variety of applications in biology, medicine, environment and catalysis. The useful properties of nanoceria are a consequence of their dual reversible oxidation states of cerium $\mathrm{Ce}^{3+} / \mathrm{Ce}^{4+}$ onto the NP surface, which allows them to act as catalysts and to mimic the activity of oxidase and peroxidase enzymes such as superoxide dismutase [2] and catalase [3]. Polymer coated nanoceria were found to have an intrinsic oxidase-like activity [4]. As compared to systems based on biocatalytic enzymes, $\mathrm{CeO}_{2} \mathrm{NPs}$ are robust, inexpensive and not susceptible to denaturation and temperature variations. Their redox properties and inherent stability provide attractive opportunities for development of new analytical devices with increased performance for field analysis [5].

Several types of inorganic NPs have been studied for their enzyme mimetic properties including $\mathrm{Fe}_{3} \mathrm{O}_{4}$ NPs [6-8], $\mathrm{Co}_{3} \mathrm{O}_{4}$ [9] and $\mathrm{CuO}$ [10] among others [11]. Ferromagnetic iron oxide NPs have been explored as materials in colorimetric assays to detect $\mathrm{H}_{2} \mathrm{O}_{2}$ and glucose [7, 12]. Previously, these particles have been used in conjunction with biomolecules (e.g. redox proteins, enzymes, antibodies) to enhance bio-stability [13, 14], facilitate electron transfer [15], enhance biocatalytic signals [16] and provide an oxygen buffering capacity for oxidase enzymes [17]. We have studied the catalytic activity of nanoceria towards $\mathrm{H}_{2} \mathrm{O}_{2}$ and have demonstrated the use of these particles as redox active material for the electrochemical [18] and colorimetric [19] detection of $\mathrm{H}_{2} \mathrm{O}_{2}$ and glucose. Nanoceria has been used as a colorimetric indicator for antioxidants activity taking advantage of color changes induced by modifications in NP surface properties on a paper platform $[20,21]$. In a recent study, we have demonstrated superior oxidase-like activity of nanoceria against catechol and dopamine as compared to tyrosinase when used in a colorimetric assay [22], and the use of these particles as catalytic amplifiers [23] and labels in bioaffinity assays [24].

Here, we propose a disposable electrochemical sensor with immobilized nanoceria on screen printed electrodes (SPE) for analyzing phenolic antioxidants. These compounds have received considerable attention due to their ability to scavenge free radicals and protect against oxidative stress thus providing health benefits $[25,26]$. A variety of antioxidant sensors with 
electrochemical detection have been reported [27-29]. A preferred sensing design is to utilize tyrosinase [30,31] or laccase enzymes [32-34] to convert the phenolic compound into its quinone derivative that can then be easily reduced and detected at a low applied potential, thus minimizing interferences. Here we describe a novel approach that utilizes inorganic biomimetic nanoceria with oxidase-like activity to replace laccase and tyrosinase enzymes in the construction of an electrochemical sensor for the detection of readily oxidizable phenolic compounds. The method is particularly applicable for analysis phenolics with orthodihydroxybenzene functionality. The sensor was fabricated on a SPE platform, which facilitates development of inexpensive detection systems and portable instrumentation for food and environmental analysis $[35,36]$. We demonstrate that nanoceria can be successfully used as electrode material for the detection of oxidizible phenolics in wine samples in a single step procedure, providing enhanced stability and comparable characteristics to previously reported electrodes which utilize the enzyme counterpart. The biomimetic sensor shows great promise as a robust tool for sensitive, rapid and cost-effective analysis of antioxidants. The sensor can find a wide range of potential applications in food chemistry and biotechnology.

\section{Materials and Methods}

\subsection{Reagents and equipment}

Cerium (IV) oxide NPs 20 wt. \% colloidal dispersion in $2.5 \%$ acetic acid with an average particle size of 10-20 nm (289744) and sodium phosphate were from Sigma Aldrich. Gallic acid (GA) was purchased from Acros, quercetin (Q) from Alfa Aesar and caffeic acid (CA) from Spectrum Chemical. Potassium phosphate monobasic was supplied from Fisher Scientific. Sodium phosphate (dibasic, anhydrous) was purchased from J.T. Baker (Phillipsburg, NJ, USA). A solution of $20 \mathrm{mM}$ potassium hexacyanoferrate (III) prepared in $0.2 \mathrm{M}$ potassium chloride was used in cyclic voltammetric experiments. Stock solutions of $10 \mathrm{mM}$ phenolic antioxidants were prepared daily before use: $\mathrm{CA}$ in ethanol, Q in acetone and GA in water. Working solutions were prepared in phosphate buffer (PBS) at $\mathrm{pH}$ 7.4. All studies were performed at a $\mathrm{pH}$ of 7.4, where the particles have the highest oxidase-like activity against phenolic compounds [22]. 
Experiments using cyclic voltammetry $(\mathrm{CV})$ were performed with an Epsilon potentiostat (BASi, West Lafayette, IN, USA). Amperometric experiments were carried out with a portable electrochemical analyzer from Dropsens (Drope-Sense $\mu 8400$ Potentiostat, Dropsens, Spain, equipped with 8 channels). All experiments were carried out using a SPE three electrode system from Dropsens (DS Dropsens DRP 110" carbon) with the nanoceria modified carbon electrode as the working electrode. Fourier Transform Infrared Spectroscopy (FTIR) spectra were recorded using a Mattson Galaxy 2020 spectrometer. FTIR samples included bare nanoceria particles and nanoceria exposed to $\mathrm{CA}$, dried for minimum $48 \mathrm{~h}$, mixed with $\mathrm{KBr}$ powder ( $\sim 5 \%$ sample in $\mathrm{KBr}$ ) and pressed into a pallet. FTIR spectra were recorded immediately. High resolution transmission electron microscopy (HR-TEM) images were taken using a high-resolution JEOL 2010 transmission electron microscope.

\subsection{Procedures}

To fabricate the nanoceria functionalized electrode a colloidal NPs suspension of $2 \%(\mathrm{w} / \mathrm{v})$ nanoceria was prepared by dispersing particles in distilled water. $3 \mu \mathrm{L}$ of this mixture was casted onto the working electrode surface of the SPE and allowed to dry for two days at room temperature until use. Electrodes were stored at room temperature with no further treatment. CV measurements were carried out in phosphate buffer solution at $\mathrm{pH} 7.4$ in the potential range from -0.4 to $0 \mathrm{~V} v s$. $\mathrm{Ag} / \mathrm{AgCl}$, at a scan rate of $100 \mathrm{mVs}^{-1}$. Amperometric experiments to obtain calibration curves were carried out at a constant applied potential of $-0.1 \mathrm{~V} v s \mathrm{Ag} / \mathrm{AgCl}$ reference electrode, with the nanoceria sensor placed vertically in a $10 \mathrm{~mL}$ electrochemical cell under constant magnetic stirring at $500 \mathrm{rpm}$. All experiments were performed at room temperature and electrodes were used for single analysis, i.e. used for the analysis of one sample, after which they were discarded. The variation in current intensity after addition of antioxidants/wine samples in the cell was measured upon signal stabilization, when the variation in current was not more than $2 \mathrm{nA} /$ minute. The data reported represent average measurements of $\mathrm{n}=3$ independent sensors fabricated using the same procedure.

\subsection{Analysis of real samples}

Two varieties of red wine (Negru Aromat and Feteasca Neagra) from Valea Calugareasca vineyard in Romania from the 2012 and 2013 harvest were tested for their antioxidant content 
with this method. These wines correspond to various maceration techniques as follows: classical maceration - V1, maceration with partial running-off of the juice -V2, maceration with use of pectolytic enzyme-V3 and maceration with addition of exogeneous tannin-V4. The wine samples were used without any pre-treatment and were added directly in the electrochemical cell containing PBS buffer at $\mathrm{pH}$ 7.4. The antioxidant potential of wine samples was explored in parallel by the nanoceria-modified electrodes and the TEAC assay for total antioxidant capacity. The antioxidant activity of the wine samples measured with the nanoceria sensors was expressed as gallic acid equivalents (GAE), obtained by interpolation of the determined amperometric currents of the sample into the calibration curve for GA. The TEAC assay was carried out as described in literature [35]. In short, a $7 \times 10^{-3} \mathrm{M}$ ABTS solution prepared in $2.5 \times 10^{-3} \mathrm{M}$ potassium persulfate was kept in the dark for 12-16 $\mathrm{h}$ at room temperature to generate ABTS radicals. This solution was then diluted 400times with water. The red wines were diluted 100 times with water. Next, $100 \mu \mathrm{L}$ of either diluted sample, Trolox or ethanol were mixed with 2.5 $\mathrm{mL}$ of ABTS radical solution and $0.4 \mathrm{ml} \mathrm{H}_{2} \mathrm{O}$ and the absorbance was measured after 3 min at $731 \mathrm{~nm}$. The results were expressed as Trolox equivalents. The measurements are expressed as mean of 3 replicate determinations.

\section{Results and Discussion}

\subsection{Electrochemical study of nanoceria functionalized sensors}

The nanoceria-modified electrodes with immobilized particles were first characterized by CV to test their behavior for the oxidation of a common antioxidant, GA (Figure 1A). To avoid any possible electrochemical oxidation process of GA, the nanoceria-induced oxidation of GA was tested in the potential range between -0.4 and $0 \mathrm{~V}$. The CVs of bare SPE carried out in PBS $(\mathrm{pH}$ 7.5 ) in the absence and in the presence of $0.5 \mathrm{mM}$ GA are similar. The CV of ceria modified SPE obtained in absence of GA showed no peaks in PBS indicating lack of electrochemical activity of these particles in the potential range tested (Figure 1 - blue). When the CV was recorded in the presence of $0.5 \mathrm{mM} \mathrm{GA}$, a reduction wave was observed at $-0.11 \mathrm{~V}$. This behavior is consistent with a fast, almost instantaneous GA oxidation by nanoceria resulting in formation of a quinoic 
compound that is then reduced electrochemically in the negative potential range starting from around $-0.05 \mathrm{~V}$ vs the $\mathrm{Ag} / \mathrm{AgCl}$ reference electrode.

\section{Figure 1}

The reduction current was proportional with the amount of GA present in solution as illustrated in Figure 2A. By increasing GA concentration from 0.05 to $0.5 \mathrm{mM}$, the reduction peak potential slightly shifted from -0.09 to $-0.11 \mathrm{~V}$ respectively. These results suggest the presence of some diffusional barriers for the oxidized GA to/from ceria surface. Figure $2 \mathrm{~B}$ shows the effect of scan rate on the intensity of the reduction current. The reduction current increased proportionally with the square root of scan rate with a correlation coefficient of $0.99(\mathrm{~N}=5)$ with a slight shift towards negative potentials, suggesting a quasi-reversible process which is diffusion controlled. This behavior is also illustrated in amperometry experiments comparing the response of bare and nanoceria-modified electrodes to successive additions of antioxidant (GA), when the electrodes are polarized at $-0.1 \mathrm{~V}$ (Figure $3 \mathrm{~A}$ ). In this case the detection limit was $1.5 \mu \mathrm{M}$ GA. The higher response of nanoceria based electrodes compared to bare electrodes towards various antioxidants, observed in amperometry experiments at low negative potentials is due to the reduction of the nanoceria-generated quinone formed as a result of catalytic oxidation by the surface-confined particles (Figure 3B). A general schematic of the oxidation/detection process is shown in Figure 3B. The reaction is ascribed to a rapid oxidation of the phenolate ion, that is likely to be present at the $\mathrm{pH}$ of the measurement environment to a semiquinone radical and then to a reactive quinone.

\section{Figure 2}

\section{Figure 3}

Scanning electron microscope (SEM) images in Figure S1 in the Supporting Information (SI) show homogenous surface coverage by nanoceria particles that coat the carbon surface on the bare SPE electrode. The X-ray elemental analysis (EDS) pattern on the modified electrode surface confirms the characteristics peaks of ceria. Moreover, in the present electrode design, the nanoceria particles oxidize the phenolic antioxidants at the phenolic ring, producing reactive semiquinone derivatives, similar to that produced by the oxidation by tyrosinase or laccase [22, 32, 37, 38]. The highest reactivity with nanoceria is expected for easily oxidizable compounds with o-dihidroxy functionality [20]. In quercetin for example the binding and reaction with 
nanoceria occurs at the dihydroxybenzene moiety. In the case of ascorbic acid which lacks the catecholate structure but presents $4 \mathrm{OH}$ groups that could bind to nanoceria, its reaction with nanoceria leads to dehydroascorbic acid as follows:

$2 \mathrm{CeO}_{2}+3 \mathrm{C}_{6} \mathrm{H}_{8} \mathrm{O}_{6} \rightarrow \mathrm{Ce}_{2} \mathrm{O}_{3}+3 \mathrm{C}_{6} \mathrm{H}_{6} \mathrm{O}_{6}+2 \mathrm{H}_{2} \mathrm{O}$

The catalytically produced quinone can form charge transfer complexes with nanoceria [20], increasing the local concentration of the reactive quinone at the electrode surface further enhancing sensitivity. FTIR analysis of nanoceria exposed to CA showed disappearance of the hydroxyl groups of CA at 1356 and $1220 \mathrm{~cm}^{-1}$ (SI, Figure S2). Therefore, the amplified electrochemical response obtained at the nanoceria electrode is due to the electrochemically active quinonic compounds that are readily reducible at a low applied potential.

We have also characterized the nanoceria-modified electrodes by $\mathrm{CV}$ to test the electrochemical behavior towards several other antioxidants, representing major polyphenolic compounds typically found in wine. Besides GA (trihydroxybenzoic acid) discussed above, we have studied catechin (a flavan-3-ol) and tannic acid (as a model for wine tannins). Ascorbic acid was also investigated as a non-phenolic antioxidant that can be purposely added to wines as per current practices. Control CVs of nanoceria electrodes showed no peaks in PBS indicating lack of electrochemical activity of these particles in the potential range tested (from -0.4 to $0.9 \mathrm{~V}$, see grey curve in Figure S3A). In the presence of antioxidants, a lower anodic current accompanied by higher reduction current in the negative potential range was observed on nanoceria electrodes, as compared to unmodified electrodes. This confirms the mechanism of nanoceria-mediated electrochemical detection of wine antioxidants discussed above. Further experiments were conducted to evaluate the analytical capability of this method by amperometry at $-0.1 \mathrm{~V}$ vs the screen printed $\mathrm{Ag} / \mathrm{AgCl}$.

\section{Figure S1}

Figure S2

Figure S3

\subsection{Analytical characteristics: sensitivity, detection limit, linear range and response time}

Figure 4 displays the calibration curves of the nanoceria sensors for GA, CA, ascorbic acid, Q and epicatechin. With addition of antioxidant, a well-defined amperometric response 
proportional with the concentration was observed. Method optimization was conducted first with respect to nanoceria loading per electrode and the magnitude of the applied potential. Enhanced sensitivity was observed for $2 \%(\mathrm{w} / \mathrm{v})$ nanoceria dispersion at a loading of $3 \mu \mathrm{l} / \mathrm{electrode}$ using GA as model analyte (SI, Figure S4A). The lower current intensity observed for high nanoceria loadings (e.g. 5\%) may be due to inactivation of the electrode surface due to formation of nanoceria aggregates blocking and electrochemical active surface of the electrode while the decrease seen for the lower amounts of nanoparticles $(0.5 \%)$ indicates lower catalytic efficiency due to fewer particles.

The sensor demonstrated comparable sensitivity in the applied potential range from -0.2 $\mathrm{V}$ to $0.05 \mathrm{~V}$ (Figure S4B) when assessed using CA. A value of $-0.1 \mathrm{~V}$ vs $\mathrm{Ag} / \mathrm{AgCl}$ was chosen for further calibration experiments to avoid potential electrochemical interferences with wine samples based on our previous studies [35]. The sensor response reached $95 \%$ of the steady-state current in $\sim 40 \mathrm{sec}$. The detection limits for the antioxidants, calculated according to $3 \sigma_{\mathrm{s}} / \mathrm{R}^{\prime}$ criteria, where $\mathrm{R}^{\prime}$ is the slope of the linear calibration curve and $\sigma_{\mathrm{s}}$ is the standard deviation of the amperometric signal of the blank solution were 15.3, 8.6 and $0.4 \mu \mathrm{M}$ for CA, Q and ascorbic acid respectively. Considering a dilution factor of 200 for wine samples, these figures would correspond to $3.06 \mathrm{mM}, 1.72 \mathrm{mM}$ and $80 \mu \mathrm{M}$ for $\mathrm{CA}, \mathrm{Q}$ and ascorbic acid respectively in wines.

\section{Figure 4}

\section{Figure S4}

The analytical characteristics are summarized in Table 1 in comparison with enzyme-based biosensors reported in literature for the detection of these analytes. Nanoceria-modified SPE electrodes provide slightly higher detection limits and linear ranges as compared to their enzyme counterparts but the concentrations detected are in the useful range for antioxidants and these sensors are far superior in terms of storage conditions, e.g. can be stored at room temperature due to the inorganic nature of the NPs catalyst. In identical conditions, control sensors in absence of particles showed no amperometric response at the applied potential of $-0.1 \mathrm{~V}$, indicating that in absence of particles, bare carbon electrodes are not able to quantitatively detect antioxidants. This difference demonstrates the role of nanoceria to catalytically form reducible quinones onto the electrode surface.

\section{Table 1}




\subsection{Study of interferences, reproducibility and stability}

The selectivity of the nanoceria electrodes against two major constituents in beverages, glucose and ethanol, is demonstrated in Figure 5A. The sensor response to both glucose and ethanol is negligible as compared to that of a wine sample or GA alone. Moreover, the sensors did not respond to a model wine solution containing $3 \mathrm{~g} . \mathrm{L}^{-1}$ tartaric acid and $12 \%$ ethanol, to a $1 \mathrm{~g} . \mathrm{L}^{-1}$ citric acid or a 0.180 g. $\mathrm{L}^{-1}$ sulphur dioxide solution. All these analytes were tested at the typical or maximum admissible concentrations in wines and were found not to interfere with the response of nanoceria sensors. Sensor reproducibility was estimated by testing the electrodes on different days following the same protocol. The relative standard deviation values for the nanoceria sensors $(n=3)$ after addition of the same concentration of GA in the range 10-500 $\mu \mathrm{M}$ was between 0.9 and $10.6 \%$. Improvement in reproducibility can be obtained by depositing the particles using an automatic procedure (e.g. inkjet printing) and by use of organic conductive linkers to create high density better-dispersed NP rich layers. The sensors are stable for months or years at room temperature as the particles are not degraded at ambient conditions, by virtue of their inorganic nature. This stability makes these particles ideal candidates for developing robust sensing devices for field deployable applications and it is one of the main advantages of this electrode design as compared with the enzymatic approach. Therefore, these sensors can be employed for single measurements and used for analysis of a large number of antioxidantcontaining samples. Determination of unknowns can be performed using a pre-recorded calibration curve obtained with similarly prepared electrodes.

\section{Figure 5}

\subsection{Real sample analysis}

To demonstrate the applicability of our method to real sample analysis, seven wines -one white and six red - were analyzed with the nanoceria electrochemical sensor. The red wines were from the same vineyard and corresponded to two varieties from 2012 and 2013 vintage, obtained by different maceration-fermentation procedures. Wine samples were added directly in the electrochemical cell and the current intensity recorded was interpolated on the calibration curve for GA to calculate the antioxidant activity. Prior these measurements, several wine dilutions (dilution factors ranging from 50 to 1000) were investigated. The best correlation between the 
currents recorded for the wine samples and the linear range of GA corresponded to a dilution factor of 200 (SI, Figure S4C), which was selected for further tests. The results, represented in Figure 5B showed a decrease in antioxidant activity in the order: Negru Aromat V1 2013> Feteasca Neagra V1 2013> Negru Aromat 2012 > white wine for wines produced by classic maceration-fermentation technology. These results also prove the possibility to evaluate the antioxidant activity of both white and red wines using the nanoceria electrochemical assay. Furthermore, the antioxidant activity of red wines and several pure compounds- expressed as GA equivalents (GAE) based on the nanoceria sensor response was compared with that determined by the conventional ABTS assay (Table 2). The antioxidant activity of the compounds tested decreased in the order $\mathrm{AA}>\mathrm{GA}>$ Quercetin $>\mathrm{CA}$, in agreement with the classification observed using a colorimetric, paper-based test that also involves nanoceria [20]. Implementation of this assay by the wine industry should include an analysis of the synergistic/antagonistic effect of ascorbic acid and sulphur dioxide, present in wine at different concentrations (at variable levels depending on the type of wine) [39] that can reduce the nanoceria-generated quinones and affect the accuracy of the phenolic signal.

Statistical analysis of results in Table 2 was performed using the Analysis ToolPak add-in in Microsoft Excel. No correlation was found between the results provided by the two methods. The differences in antioxidant activities of various samples recorded with the two methods originate from their basic principles - oxidizability by nanoceria versus scavenging of ABTS ${ }^{+}$ radical. Such differences are a common occurrence among assays of antioxidant capacity based on different mechanisms [20]. Moreover, these differences are an advantage in devices such etongues or arrays which are based on a set of sensors with different selectivity towards varying analytes or sample types. In this regard, it is important to note here that while the ABTS assay has shown relatively small differences between the wines obtained by various maceration techniques, the nanoceria sensors were much more sensitive to differences between samples (Table 2). Consequently, nanoceria sensors might be a useful tool for differentiating wines produced by various winemaking techniques, and be used to define a 'fingerprint' of particular wine types.

\section{Table 2.}




\section{Conclusion}

In conclusion, we have demonstrated the feasibility of using nanoceria particles as oxidase mimetics for the detection of wine antioxidants using electrochemical methodologies. As shown, the nanoceria based SPE sensors provide rapid one step detection of antioxidants and demonstrate functionality for the analysis of wine samples. Stability, robustness and ease-ofpreparation of these sensors offer unique advantages. The sensors provide an alternative to similar tests based on natural enzymes that often suffer from limited long-term stability, high cost and inability to function outside their optimum conditions. The electrochemical detection avoids the disadvantages of optical tests that may suffer from interferences from highly colored samples such as red wine and enable a more sensitive detection. The developed nanoceria SPE electrodes are low cost, stable and disposable making them appealing for rapid field detection and screening purposes of antioxidant rich samples. These modified electrodes could be a useful tool in evaluating different varieties of wines and be used to complement or replace classic antioxidant tests. Differences in response captured with nanoceria-modified electrodes could potentially be included in an e-tongue device assisting with analytical fingerprinting of wines of many varieties.

\section{Acknowledgements}

This work was supported in part by a grant of the Romanian National Authority for Scientific Research, CNDI - UEFISCDI, project number PN-II-PT-PCCA-2011-3.1-1809. We are grateful to Dr. Eng. Aurelia Tudorache from the ICDVV Valea Calugareasca, Romania, for providing the wine samples. This material is based upon work supported in part by the National Science Foundation under Grant No. 0954919. Any opinions, findings, and conclusions or recommendations expressed in this material are those of the author(s) and do not necessarily reflect the views of the National Science Foundation.

\section{References}

[1] Karakoti A, Singh S, Dowding JM, Seal S, Self WT. Redox-active radical scavenging nanomaterials. Chem Soc Rev. 2010;39:4422-32.

[2] Korsvik C, Patil S, Seal S, Self WT. Superoxide dismutase mimetic properties exhibited by vacancy engineered ceria nanoparticles. Chem Commun. 2007:1056-8. 
[3] Pirmohamed T, Dowding JM, Singh S, Wasserman B, Heckert E, Karakoti AS, et al. Nanoceria exhibit redox state-dependent catalase mimetic activity. Chem Commun. 2010;46:2736-8.

[4] Asati A, Santra S, Kaittanis C, Nath S, Perez JM. Oxidase-Like Activity of Polymer-Coated Cerium Oxide Nanoparticles. Angew Chem Int Edit. 2009;48:2308-12.

[5] Sharpe E, Andreescu S. Portable Nanoparticle Based Sensors for Antioxidant Analysis. Methods Mol Biol. 2015;1208:221-31.

[6] Gao LZ, Zhuang J, Nie L, Zhang JB, Zhang Y, Gu N, et al. Intrinsic peroxidase-like activity of ferromagnetic nanoparticles. Nat Nanotechnol. 2007;2:577-83.

[7] Su L, Feng J, Zhou XM, Ren CL, Li HH, Chen XG. Colorimetric Detection of Urine Glucose Based ZnFe2O4 Magnetic Nanoparticles. Anal Chem. 2012;84:5753-8.

[8] Wei H, Wang E. Fe3O4 magnetic nanoparticles as peroxidase mimetics and their applications in $\mathrm{H} 2 \mathrm{O} 2$ and glucose detection. Anal Chem. 2008;80:2250-4.

[9] Mu JS, Wang Y, Zhao M, Zhang L. Intrinsic peroxidase-like activity and catalase-like activity of Co3O4 nanoparticles. Chem Commun. 2012;48:2540-2.

[10] Chen W, Chen J, Liu AL, Wang LM, Li GW, Lin XH. Peroxidase-Like Activity of Cupric Oxide Nanoparticle. Chemcatchem. 2011;3:1151-4.

[11] Xie JX, Zhang XD, Wang H, Zheng HZ, Huang YM. Analytical and environmental applications of nanoparticles as enzyme mimetics. Trac-Trend Anal Chem. 2012;39:114-29.

[12] Mita DG, Attanasio A, Arduini F, Diano N, Grano V, Bencivenga U, et al. Enzymatic determination of BPA by means of tyrosinase immobilized on different carbon carriers. Biosensors \& Bioelectronics. 2007;23:60-5.

[13] Saha S, Arya SK, Singh SP, Sreenivas K, Malhotra BD, Gupta V. Nanoporous cerium oxide thin film for glucose biosensor. Biosensors \& Bioelectronics. 2009;24:2040-5.

[14] Kaushik A, Solanki PR, Ansari AA, Ahmad S, Malhotra BD. A nanostructured cerium oxide film-based immunosensor for mycotoxin detection. Nanotechnology. 2009;20.

[15] Xiao XL, Luan QF, Yao X, Zhou KB. Single-crystal CeO2 nanocubes used for the direct electron transfer and electrocatalysis of horseradish peroxidase. Biosensors \& Bioelectronics. 2009;24:2447-51.

[16] Njagi J, Chernov MM, Leiter JC, Andreescu S. Amperometric Detection of Dopamine in Vivo with an Enzyme Based Carbon Fiber Microbiosensor. Anal Chem. 2010;82:989-96.

[17] Njagi J, Ispas C, Andreescu S. Mixed ceria-based metal oxides biosensor for operation in oxygen restrictive environments. Anal Chem. 2008;80:7266-74.

[18] Ispas C, Njagi J, Cates M, Andreescu S. Electrochemical studies of ceria as electrode material for sensing and biosensing applications. J Electrochem Soc. 2008;155:F169-F76.

[19] Ornatska M, Sharpe E, Andreescu D, Andreescu S. Paper Bioassay Based on Ceria Nanoparticles as Colorimetric Probes. Anal Chem. 2011;83:4273-80.

[20] Sharpe E, Frasco T, Andreescu D, Andreescu S. Portable ceria nanoparticle-based assay for rapid detection of food antioxidants (NanoCerac). Analyst. 2013;138:249-62. 
[21] Sharpe E, Bradley R, Frasco T, Jayathilaka D, Marsh A, Andreescu S. Metal oxide based multisensor array and portable database for field analysis of antioxidants. Sensor Actuat BChem. 2014;193:552-62.

[22] Hayat A, Cunningham J, Bulbul G, Andreescu S. Evaluation of the oxidase like activity of nanoceria and its application in colorimetric assays. Anal Chim Acta. 2015;885:140-7.

[23] Hayat A, Andreescu S. Nanoceria Particles As Catalytic Amplifiers for Alkaline Phosphatase Assays. Anal Chem. 2013;85:10028-32.

[24] Bulbul G, Hayat A, Andreescu S. A generic amplification strategy for electrochemical aptasensors using a non-enzymatic nanoceria tag. Nanoscale. 2015;7:13230-8.

[25] Carrizzo A, Forte M, Damato A, Trimarco V, Salzano F, Bartolo M, et al. Antioxidant effects of resveratrol in cardiovascular, cerebral and metabolic diseases. Food Chem Toxicol. 2013;61:215-26.

[26] Corder R, Mullen W, Khan NQ, Marks SC, Wood EG, Carrier MJ, et al. Red wine procyanidins and vascular health. Nature. 2006;444:566-.

[27] Prehn R, Gonzalo-Ruiz J, Cortina-Puig M. Electrochemical Detection of Polyphenolic Compounds in Foods and Beverages. Curr Anal Chem. 2012;8:472-84.

[28] Arribas AS, Martinez-Fernandez M, Chicharro M. The role of electroanalytical techniques in analysis of polyphenols in wine. Trac-Trend Anal Chem. 2012;34:78-96.

[29] Vasilescu A, Sharpe E, Andreescu S. Nanoparticle-Based Technologies for the Detection of Food Antioxidants. Curr Anal Chem. 2012;8:495-505.

[30] Abhijith KS, Kumar PVS, Kumar MA, Thakur MS. Immobilised tyrosinase-based biosensor for the detection of tea polyphenols. Anal Bioanal Chem. 2007;389:2227-34.

[31] Andreescu S, Sadik OA. Correlation of analyte structures with biosensor responses using the detection of phenolic estrogens as a model. Anal Chem. 2004;76:552-60.

[32] Di Fusco M, Tortolini C, Deriu D, Mazzei F. Laccase-based biosensor for the determination of polyphenol index in wine. Talanta. 2010;81:235-40.

[33] Gil DMA, Rebelo MJF. Evaluating the antioxidant capacity of wines: a laccase-based biosensor approach. Eur Food Res Technol. 2010;231:303-8.

[34] Penu R, Vasilescu I, Eremia SAV, Gatea F, Radu GL, Litescu SC. Development of a nanocomposite system and its application in biosensors construction. Cent Eur $\mathrm{J}$ Chem. 2013;11:968-78.

[35] Avramescu A, Andreescu S, Noguer T, Bala C, Andreescu D, Marty JL. Biosensors designed for environmental and food quality control based on screen-printed graphite electrodes with different configurations. Anal Bioanal Chem. 2002;374:25-32.

[36] Bulbul G, Hayat A, Andreescu S. Portable Nanoparticle-Based Sensors for Food Safety Assessment. Sensors-Basel. 2015;15:30736-58.

[37] Elkaoutit M, Naranjo-Rodriguez I, Temsamani KR, La Vega MD, De Cisneros JLH. Dual laccase-tyrosinase based sonogel-carbon biosensor for monitoring polyphenols in beers. J Agr Food Chem. 2007;55:8011-8. 
[38] Diaconu M, Litescu SC, Radu GL. Laccase-MWCNT-chitosan biosensor-A new tool for total polyphenolic content evaluation from in vitro cultivated plants. Sensor Actuat B-Chem. 2010;145:800-6.

[39] Abou Samra M, Chedea VS, Economou A, Calokerinos A, Kefalas P. Antioxidant/prooxidant properties of model phenolic compounds: Part I. Studies on equimolar mixtures by chemiluminescence and cyclic voltammetry. Food Chem. 2011;125:622-9.

[40] Fernandex-Pachon MSV, D.; Garcia-Parrilla, M.C.; Troncoso, A.M. Antioxidant activity of wines and relation with their polyphenolic composition. Analytical Methods. 2004;513:113-8.

[41] Davalos AG-C, Carmen; Bartolome, Begona. Extending Applicability of the Oxygen Radical Absorbance Capacity (ORAC-Fluorescein) Assay. J Agric Food Chem. 2004;52:48-54. 


\section{Captions for the Figures}

Figure 1. CV for bare SPE (black) and nanoceria - modified SPE (blue) in recorded in 0.1 M PBS (pH 7.5) and the correspondent CVs of bare SPE (red) and nanoceria modified SPE (green) obtained in PBS (pH 7.5) in the presence of $0.5 \mathrm{mM}$ gallic acid (scan rate $100 \mathrm{mV} / \mathrm{s}$ ).

Figure 2. (A). CVs of nanoceria - modified SPE recorded in 0.1 M PBS (pH 7.5) containing $0.05 ; 0.1 ; 0.25$ and $0.5 \mathrm{mM} \mathrm{GA}$ at a scan rate of $0.1 \mathrm{~V} / \mathrm{s})$ and (B). CVs of nanoceria - modified SPE recorded in 0.1 M PBS (pH 7.5) at scan rates of 50, 100, 150 and $200 \mathrm{mV} / \mathrm{s}$, respectively. Inset: the plot of reduction current $v s$ square root of scan rate.

Figure 3. (A) Amperometric response of bare (black) and nanoceria modified SPE (red) to successive additions of GA (inset: linear range of the calibration curve). (B) Proposed mechanism showing nanoceria-induced oxidation of GA with measurement of the electrochemical reduction of the catalytically generated quinone at $-0.1 \mathrm{~V} v s$ the $\mathrm{Ag} / \mathrm{AgCl} \mathrm{SPE}$ electrode.

Figure 4. Dependency of electrochemical response with the concentration for: (A) GA, (B) caffeic acid, (C) quercetin, (D) ascorbic acid and ( E) epicatechin. Amperometry experiments with nanoceria modified electrodes were carried out at $-0.1 \mathrm{~V}$ vs $\mathrm{Ag} / \mathrm{AgCl}$ with $\mathrm{n}=3$ independently prepared electrodes for each concentration. Inset: linear range.

Figure 5. A. Effect of interfering compounds of glucose $(150 \mathrm{uM})$, and ethanol (1\%), often present in beverages as compared to sensor response to GA and a wine sample. B. Demonstration of antioxidant activity calculation: the analytical signal of wines, diluted 200-fold and analyzed with the nanoceria sensors are interpolated on the standard calibration curve for GA in order to derive the concentrations of GA for each wine. The antioxidant activity of wines expressed as GAE is then calculated by conversion from logarithmic to normal concentration units (mmol. $\left.\mathrm{L}^{-1}\right)$ and multiplication by the dilution factor. 


\section{Tables}

Table 1: Analytical characteristics of the nanoceria sensors for the detection of phenolic antioxidants and comparison with enzyme based biosensors reported in literature.

\begin{tabular}{|c|c|c|c|c|c|c|}
\hline $\begin{array}{c}\text { Sensor configuration / } \\
\text { electrode material }\end{array}$ & $\begin{array}{c}\text { E }_{\text {appl }}(\mathrm{V} \text { vs. } \\
\text { Ag/AgCl) }\end{array}$ & Analyte & $\begin{array}{c}\text { Linear range } \\
(\mu \mathrm{M})\end{array}$ & $\begin{array}{l}\text { LOD } \\
(\mu \mathrm{M})\end{array}$ & $\begin{array}{c}\text { Response time / } \\
\text { storage details }\end{array}$ & $\begin{array}{c}\text { Refere } \\
\text { nces }\end{array}$ \\
\hline $\begin{array}{l}\text { Tyrosinase immobilized in a } \\
\text { carbon paste }\end{array}$ & -0.15 & Q & $1-25$ & 1.5 & $3 \mathrm{~min} / \mathrm{n} . \mathrm{a}$ & [31] \\
\hline $\begin{array}{l}\text { Laccase immobilized in } \\
\text { polyzetidine prepolymer and } \\
\text { SWCNTs }\end{array}$ & -0.1 & GA & $0.53-96$ & - & $\begin{array}{c}\text { n.a / } 10 \text { days } \\
\text { storage at } 40^{\circ} \mathrm{C}\end{array}$ & {$[23]$} \\
\hline $\begin{array}{l}\text { Tyrosinase and laccase } \\
\text { immobilized in sonogel- } \\
\text { carbon }\end{array}$ & -0.2 & $\begin{array}{l}\text { GA } \\
\text { CA }\end{array}$ & $\begin{array}{c}0.1-15 \\
0.01-2.0\end{array}$ & $\begin{array}{c}0.19 \\
0.026\end{array}$ & $\begin{array}{l}14 \mathrm{sec} / 80 \% \\
\text { activity after } 3 \\
\text { weeks at } 40^{\circ} \mathrm{C}\end{array}$ & [30] \\
\hline $\begin{array}{l}\text { Laccase immobilized in } \\
\text { chitosan/ MWCNTs }\end{array}$ & -0.2 & $\begin{array}{l}\mathrm{GA} \\
\mathrm{CA}\end{array}$ & $\begin{array}{c}0.79-2.1 \\
0.73-1.05\end{array}$ & $\begin{array}{c}- \\
0.275\end{array}$ & $2 \mathrm{~min} / \mathrm{n} \cdot \mathrm{a}$ & [31] \\
\hline $\begin{array}{l}\text { Nanoceria SPE } \\
\text { Reductive detection of } \\
\text { nanoceria generated quinone }\end{array}$ & -0.1 & $\begin{array}{c}\text { GA } \\
\text { CA } \\
\text { Q } \\
\text { AA }\end{array}$ & $\begin{array}{c}0.03-0.2 \\
0.05-0.2 \\
0.02-0.2 \\
0.0005-0.02\end{array}$ & $\begin{array}{l}1.5 \\
15.3 \\
8.6 \\
0.4\end{array}$ & $\begin{array}{c}40 \mathrm{sec} / \\
\text { Room } \\
\text { temperature } \\
\text { Single use }\end{array}$ & $\begin{array}{l}\text { This } \\
\text { work }\end{array}$ \\
\hline
\end{tabular}

SPE - screen printed electrode; SWCNTs - single wall carbon nanotubes. MWCNTs - multi wall carbon nanotubes Tyr-Tyrosinase; GA-gallic acid; Q-quercetin; CA-caffeic acid; AA-ascorbic acid; n.a - not available 


\section{Table 2:}

Antioxidant activities of red wines and several relevant compounds assessed in parallel by the nanoceria-based electrochemical assay and by the classic TEAC assay.

\begin{tabular}{|l|c|c|}
\hline \multicolumn{1}{|c|}{ Sample } & Nanoceria SPE $^{\mathrm{a}}$ & $\begin{array}{c}\text { TEAC test based on } \\
\text { ABTS }^{\mathrm{b}}\end{array}$ \\
\hline Caffeic acid & $0.73 \pm 0.05$ & $5.02 \pm 0.37$ \\
\hline Quercetin & $1.59 \pm 0.10$ & $5.82 \pm 0.27$ \\
\hline Epicatechin & $3.66 \pm 1.64$ & $7.81 \pm 0.49$ \\
\hline Ascorbic acid & $15.77 \pm 1.47$ & $4.05 \pm 0.20$ \\
\hline Negru Aromat 2012 & $2.80 \pm 0.54$ & $25.00 \pm 0.70$ \\
\hline Negru Aromat V1, 2013 & $5.07 \pm 0.79$ & $13.99 \pm 1.10$ \\
\hline Negru Aromat V2, 2013 & $4.29 \pm 0.19$ & $13.00 \pm 0.66$ \\
\hline Feteasca Neagra V1, 2013 & $3.49 \pm 0.50$ & $6.57 \pm 0.32$ \\
\hline Feteasca Neagra V3, 2013 & $4.58 \pm 0.58$ & $5.89 \pm 0.48$ \\
\hline Feteasca Neagra V4, 2013 & $4.86 \pm 0.80$ & $7.11 \pm 0.85$ \\
\hline
\end{tabular}

${ }^{\mathrm{a}}$ Expressed as mmol.g ${ }^{-1}$ GAE (for antioxidants) and mmol.L ${ }^{-1}$ GAE (for red wines); ${ }^{b}$ Expressed as mmol.g ${ }^{-1}$ TEAC (for antioxidants) and as mmol.L ${ }^{-1}$ TEAC (for red wines). Values compare well to previously published ORAC and ABTS values for red wines and polyphenols [40, 41]. 


\section{Figures}

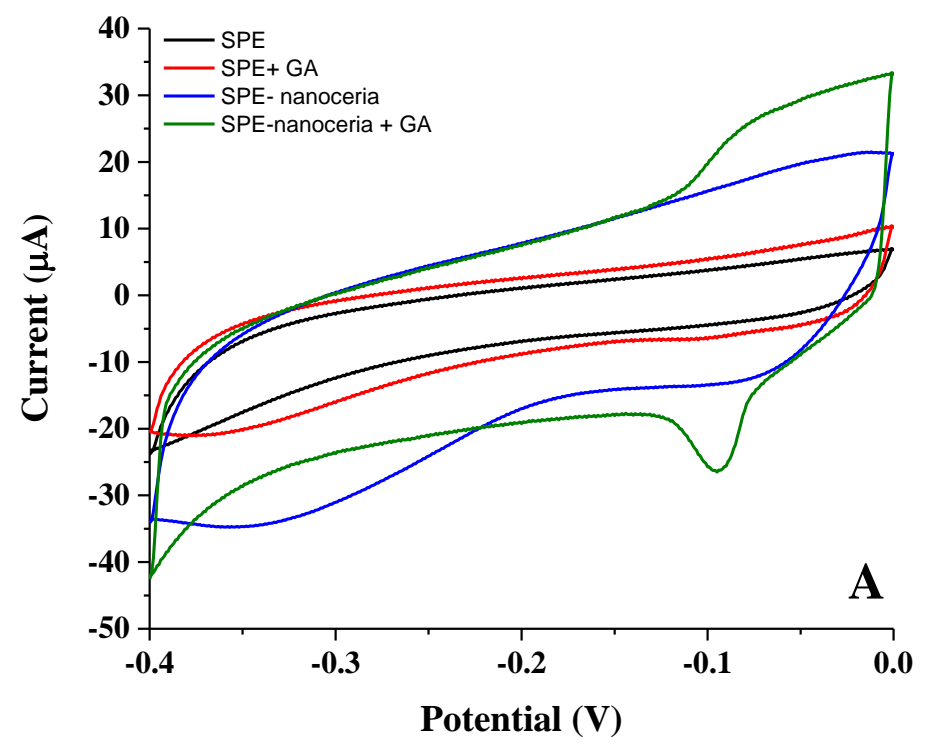

Figure 1 

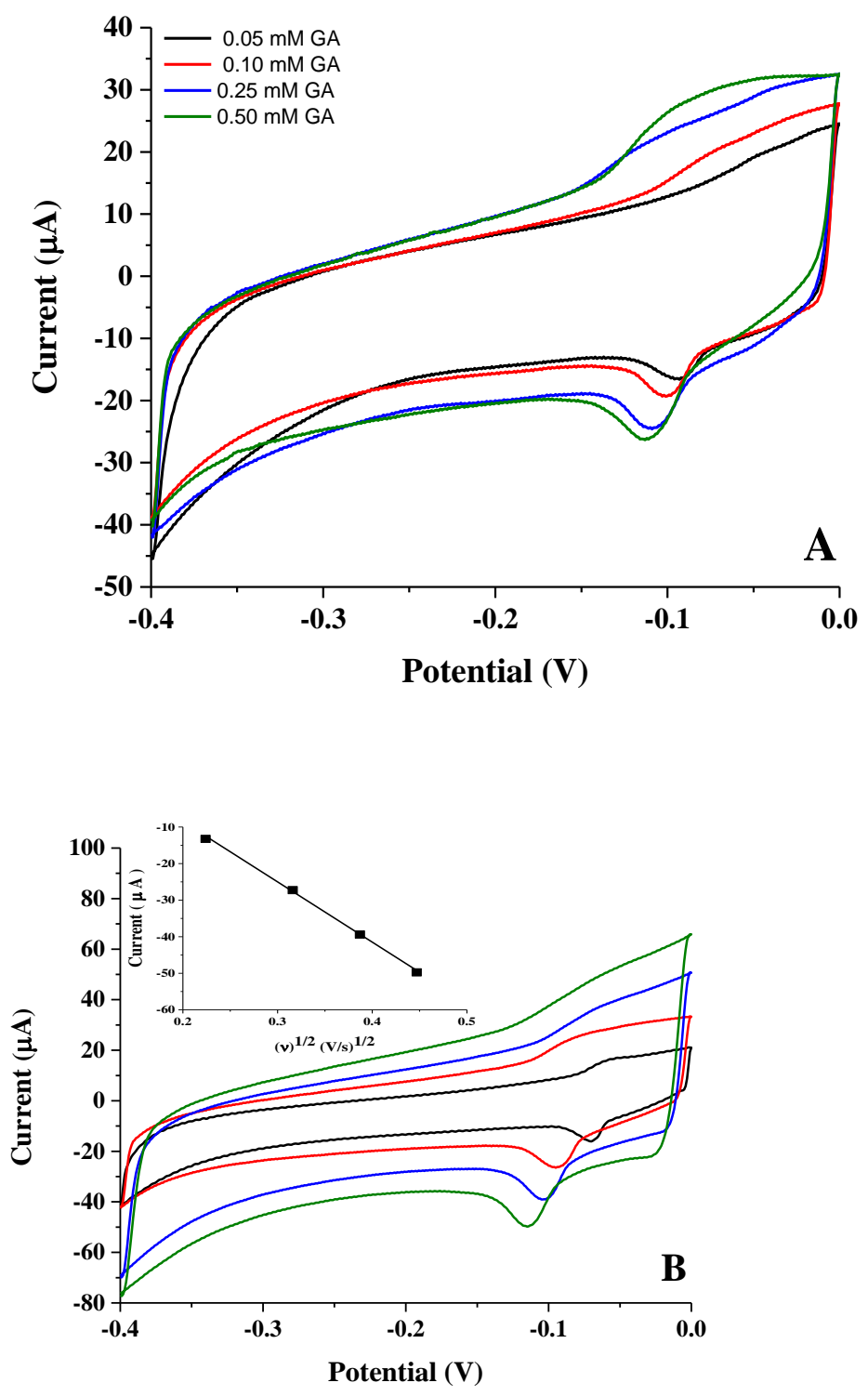

Figure 2 


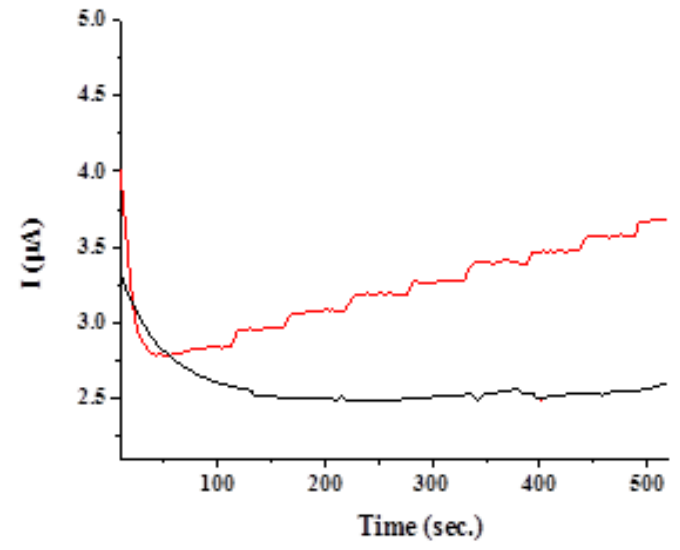

A

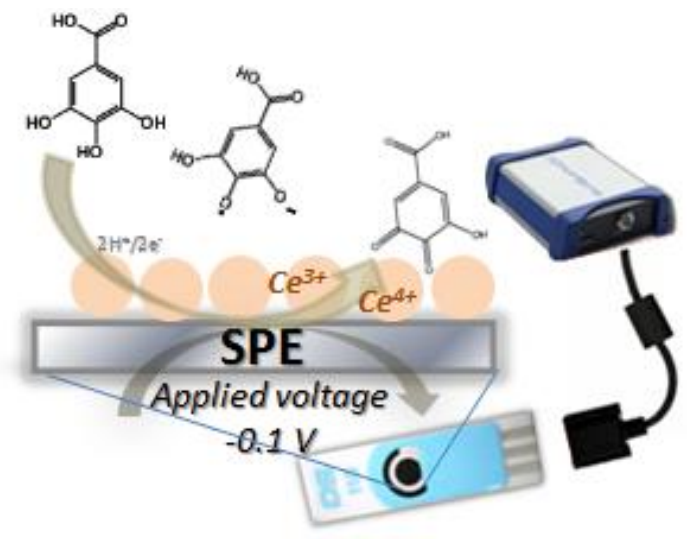

B

Figure 3 

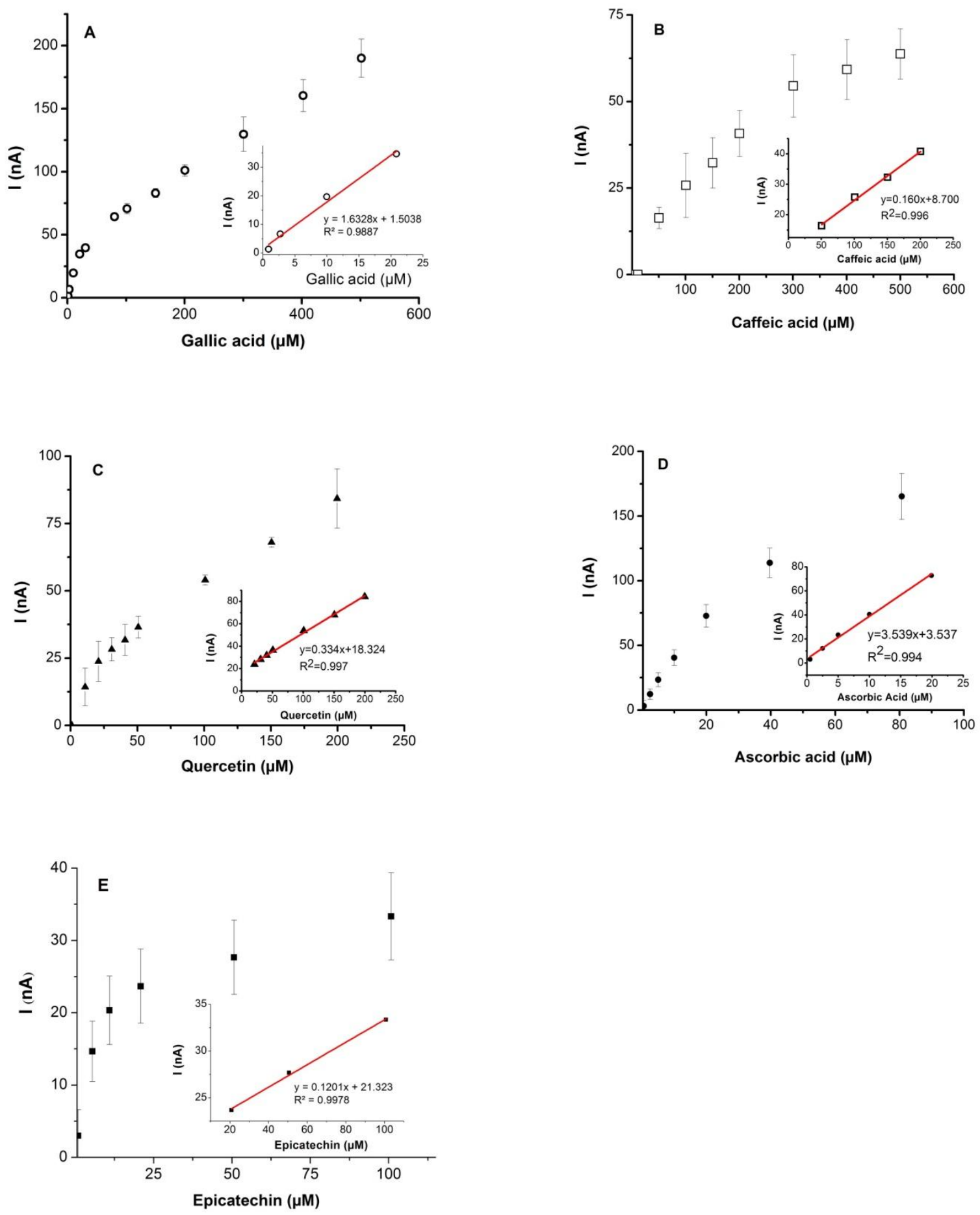

Figure 4 


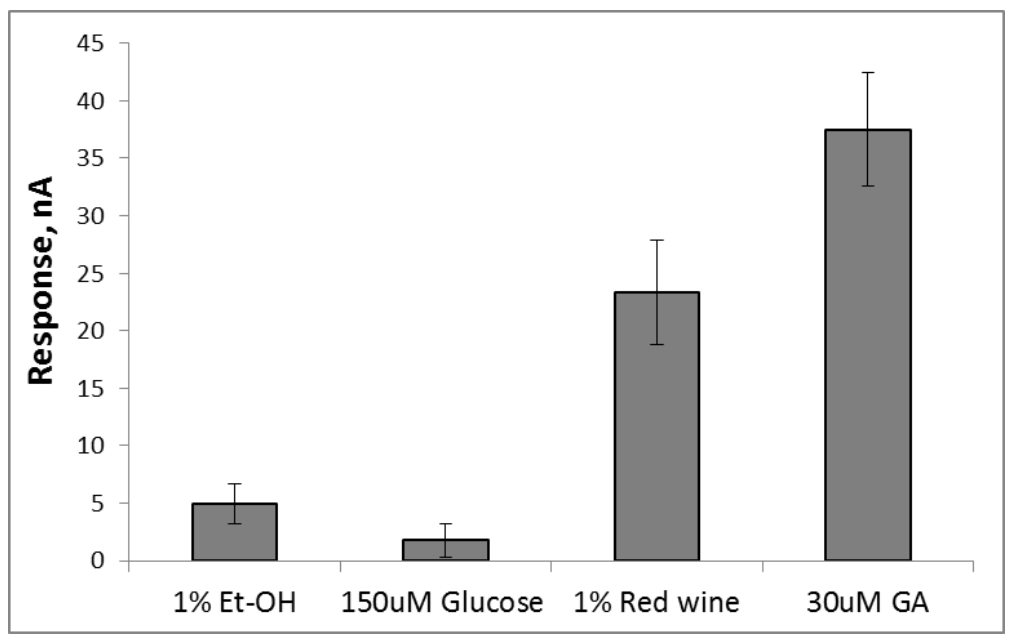

A

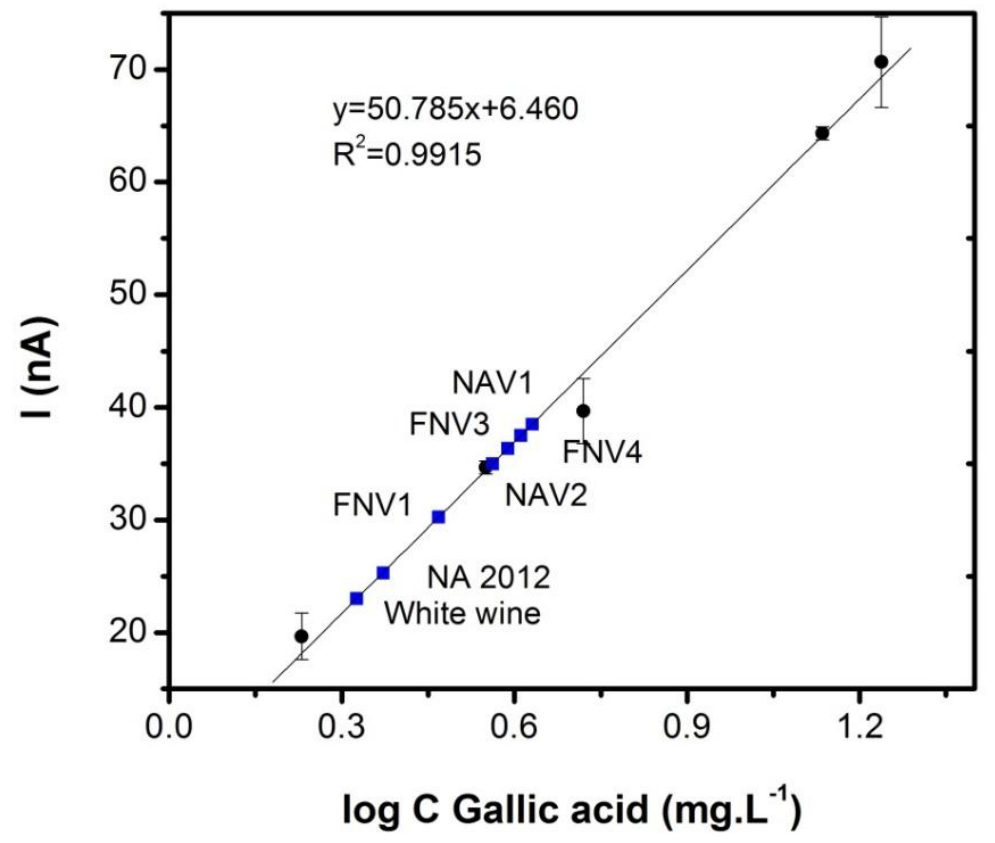

B

Figure 5 
Graphical abstract

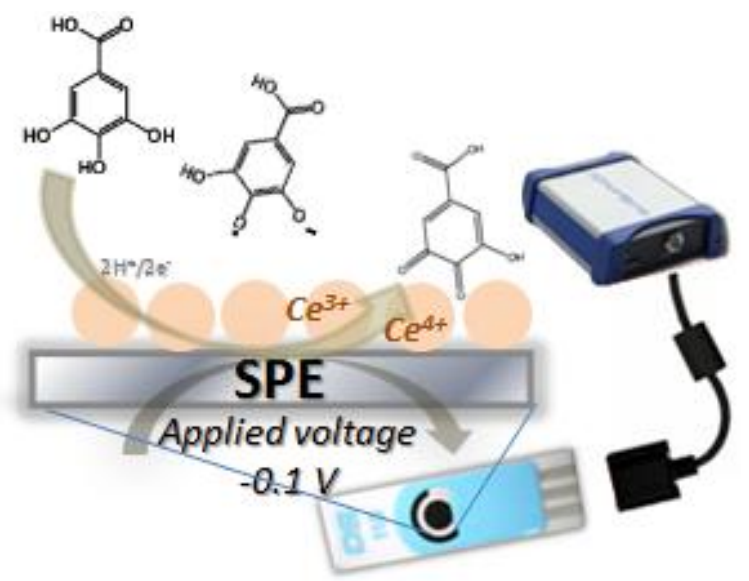

